\title{
Electron capture into few-electron heavy ions: Independent particle model
}

\author{
A. Surzhykov ${ }^{1, a}$, U.D. Jentschura ${ }^{1}$, T. Stöhlker ${ }^{2}$, and S. Fritzsche ${ }^{1,2}$ \\ 1 Max-Planck-Institut für Kernphysik, 69029 Heidelberg, Germany \\ 2 Gesellschaft für Schwerionenforschung (GSI), 64291 Darmstadt, Germany
}

Received 23 July 2007 / Received in final form 17 August 2007

Published online 21 September 2007 - (C) EDP Sciences, Società Italiana di Fisica, Springer-Verlag 2007

\begin{abstract}
We apply the density matrix theory to re-investigate the radiative electron capture into heavy ions with one valence electron. Attention has been paid particularly to the magnetic sublevel population of the residual ions, as described in terms of alignment parameters. Simple method, based on an independent particle model, which takes into account the Pauli principle, is proposed for evaluating the alignment of the excited ionic states. By making use of this method, detailed calculations are performed for electron capture into (initially) hydrogen-like and lithium-like europium, gold and uranium ions, and are compared with the results of the multiconfiguration Dirac-Fock approach.As seen from the calculations and from the comparison with available experimental results, the independent particle model provides a good estimate for the alignment parameters of few-electron heavy ions. Therefore, our simple model may help to understand the basic properties of the X-ray emission from heavy, few-electron ions without the need for invoking sophisticated MCDF calculations.
\end{abstract}

PACS. 31.25.Jf Electron correlation calculations for atoms and ions: excited states - 31.30.Jv Relativistic and quantum electrodynamic effects in atoms and molecules - 34.70.+e Charge transfer - 34.80.Lx Electron-ion recombination and electron attachment

\section{Introduction}

During the last decades, energetic collisions of highlycharged ions with atoms and free electrons have been explored intensively by using accelerators, storage rings, or electron beam ion trap (EBIT) facilities. Many processes that occur in these collisions hereby result in the production of excited ionic states. Since a preferred direction is defined for the overall system both, in the storage rings (due to the ion beam) as well as the EBIT experiments (due to the electron beam), the excited ions are often aligned with respect to this direction. This alignment, i.e. the unequal population of the magnetic sublevels with different modulus of the quantum number $\left|M_{J}\right|$, is known to modify significantly the properties of the subsequent radiative decay. The effect of a non-zero alignment can be seen, for example, from the strongly anisotropic angular distribution and the non-zero linear polarization of characteristic X-ray photons [1-4]. A great number of case studies have therefore been performed in order to explore the angular and polarization properties of the subsequent decay and to derive the alignment of highly-charged ions, following the ion-atom (or ion-electron) collisions. For the electron-impact excitation of few-electron ions, for instance, these theoretical [5-8] and experimental [9-12]

\footnotetext{
a e-mail: surz@mpi-hd.mpg.de
}

studies had a strong impact upon the diagnostics of hightemperature laboratory and astrophysical plasmas as well as for probing relativistic effects in simple atomic systems. Much attention has been paid also onto the magnetic sublevel population of the ionic states as produced by the inner-shell (impact) ionization, for which detailed calculations have been performed both, within the nonrelativistic $[2,13,14]$ as well as the relativistic distorted-wave theory $[15,16]$.

Beside the impact with ions or free electrons, an alignment of the residual ions may occur also due to a number of charge transfer reactions. For example, the production of aligned (or even oriented) ions has been studied in details for the dielectronic recombination of multiple and highly-charged ions [17-20] as well as the nonradiative electron capture [21,22]; in the latter case, a series of measurements has been carried out by using beam-foil techniques. During recent years, moreover, special emphasis was placed on the radiative electron capture (REC) into excited states of high- $Z$, few-electron ions. In order to probe the alignment caused by REC, the angular properties of the characteristic X-ray emission from heavy projectiles have been studied at the GSI storage ring in Darmstadt. Most recent experiments have dealt especially with the subsequent decay of the hydrogen-like $\mathrm{U}^{91+}$ and helium-like $\mathrm{U}^{90+}$ uranium ions [19,24-26] as produced by the radiative recombination of the initially bare or, 
respectively, hydrogen-like ions. From the (measured) angular distribution of the Lyman- $\alpha_{1}\left(2 p_{3 / 2} \rightarrow 1 s\right)$ and $\mathrm{K} \alpha_{1}$ $\left(1 s 2 p_{3 / 2} \rightarrow 1 s^{2}\right)$ photons and, hence, the alignment of the excited ions, valuable information was obtained not only about the dynamics of the electron capture processes $[25$, 26 ] but also about the multipole mixing in heavy atomic systems $[24,27]$.

In the theoretical analysis of the experiments, a "twostep" approach is usually applied, in which the creation and subsequent decay of the excited ionic states occur as two independent steps, well separated in time. Two-step calculations have first been performed by Scofield [23] for the REC into bare and hydrogen-like titanium and iron ions and have been extended later successfully towards the high- $Z$ domain [27-31]. However, while the capture into (initially) bare ions can be treated today quite rigorously by applying exact relativistic bound and continuum (electronic) wavefunctions, some approximate methods are required to evaluate the two-electron states. Apart from the multiconfiguration Dirac-Fock (MCDF) method, which provides a systematic approach to the electronelectron interaction effects $[29,30]$, the REC into (initially) hydrogen-like uranium ions $\mathrm{U}^{90+}$ can still be understood within the independent particle model [31]. This model, which takes the Pauli principle into account, should be well justified for the REC of heavy ions since the interelectronic effects are much weaker in the high- $Z$ domain than the electron-nucleus interaction. Another great advantage of the (effective) one-particle approach is, that it enables one to avoid the computationally expensive and usually non-trivial MCDF calculations. The cross sections and alignment parameters, obtained within this approach, can be therefore applied for most experiments on the X-ray emission from heavy, few-electron ions, for which precise data are often not (yet) required.

In the present paper, we generalize the effective oneparticle approach for studying the electron recombination into the excited states of ions with a single valence electron outside of closed shell. We argue that such a generalization can be done most naturally by employing irreducible tensors. When combined with the density matrix approach, these tensors describe the (degree of) alignment of the excited ionic states and, hence, can be used directly to derive the angular and polarization properties of the subsequent decay photons. In Section 2.1, therefore, we recall the general expressions for the (statistical) tensors of the excited states of few-electron ions following a radiative electron capture process. In particular, we show that any analysis of these tensors can be traced back to the many-electron matrix elements which describe the freebound electron transition under the simultaneous photon emission. The evaluation of the many-electron transition amplitudes within the independent particle approach is presented in Section 2.2. With the help of this approach, we are then able to represent the alignment parameters for the many-electron ions in terms of their one-electron analogs. In order to analyze the range of validity for such a single-particle approximation, detailed calculations are performed for the alignment of excited ionic states follow- ing the $L$ - and $M$-shell electron recombination into initially hydrogen-like and lithium-like europium, gold and uranium projectiles. The results of the (one-electron) calculations are presented in Section 3.1 and are compared with the predictions from the MCDF approach. From the comparison, it is found that an effective one-particle approach is valid for the electron capture into the excited levels of high- $Z$, few-electron ions. Moreover, in Section 3.2 we demonstrate that apart from the reasonable estimate of the alignment parameters of the excited (many-electron) ionic states, our generalized approach may help in understanding the features of the subsequent radiative decay. In particular, we consider the angular distribution of the $\mathrm{K} \alpha_{1}$ radiation from the helium-like uranium ions $\mathrm{U}^{90+}$, following the REC, for which a series of experiments have recently been performed at the GSI storage ring. When comparing our calculations with the experimental data available and the previous many-electron results, we argue that the simple one-particle approach is well suited for a - fast and reliable - analysis of the X-ray emission. This applies especially for the present level of experimental accuracy where neither the fine structure of the excited states nor the anisotropy of the subsequent photon emission is resolved to a high degree. Finally, a brief summary of these results and an outlook are given in Section 4 .

\section{Theory}

\subsection{General expression for the alignment of the excited ionic states}

Not much has to be said about the basic formalism for studying the alignment of many-electron heavy ions following an electron capture process. Recently, this formalism, based on the density matrix approach [2], has been discussed by us in a series of papers $[29,30,32]$. In particular, we argued that the magnetic sublevel population of the excited ionic states can be described most naturally in terms of the so-called statistical tensors:

$$
\begin{aligned}
\rho_{k 0}\left(\alpha_{f} J_{f}\right)= & \frac{32 \pi^{3}}{2 J_{i}+1} \sum_{L p} \sum_{J J^{\prime} \kappa \kappa^{\prime}}\left[l, l^{\prime}, j, j^{\prime}, J, J^{\prime}\right]^{1 / 2} \\
& \times(-1)^{J_{i}+L-J_{f}+J-J^{\prime}-1 / 2}\left\langle l 0 l^{\prime} 0 \mid k 0\right\rangle \\
& \times\left\{\begin{array}{ccc}
j & j^{\prime} & k \\
l^{\prime} & l & 1 / 2
\end{array}\right\}\left\{\begin{array}{ccc}
j & j^{\prime} & k \\
J^{\prime} & J & J_{i}
\end{array}\right\}\left\{\begin{array}{ccc}
J & J^{\prime} & k \\
J_{f} & J_{f} & L
\end{array}\right\} \\
& \times\left\langle\left(\alpha_{i} J_{i}, \epsilon l j\right) J\left\|H_{\gamma}(p L)\right\| \alpha_{f} J_{f}\right\rangle \\
& \times\left\langle\left(\alpha_{i} J_{i}, \epsilon l^{\prime} j^{\prime}\right) J^{\prime}\left\|H_{\gamma}(p L)\right\| \alpha_{f} J_{f}\right\rangle^{*},
\end{aligned}
$$

if both, the incident electron and initial ion are assumed to be unpolarized and the recombination X-ray photons remain unobserved. Here, $\left[l_{a}, l_{b}, \ldots\right]=\left(2 l_{a}+1\right)\left(2 l_{b}+1\right) \ldots$, $\left\langle l 0 l^{\prime} 0 \mid k 0\right\rangle$ is the Clebsch-Gordan coefficient, $J_{i}$ and $J_{f}$ are the total angular momenta of the ion before and after the recombination process, and

$$
\begin{aligned}
\left\langle\left(\alpha_{i} J_{i}, \epsilon l j\right) J\right. & \left.\left\|H_{\gamma}(p L)\right\| \alpha_{f} J_{f}\right\rangle=i^{-l} \mathrm{e}^{-i \Delta_{\kappa}} \\
& \times\left\langle\left(\alpha_{i} J_{i}, \epsilon l j\right) J\left\|\sum_{n} \boldsymbol{\alpha}_{n} \cdot \boldsymbol{A}_{L, n}^{p}\right\| \alpha_{f} J_{f}\right\rangle
\end{aligned}
$$


is the reduced matrix element for the free-bound electron transition under the simultaneous emission of a photon with angular momentum $L$ and parity $(-1)^{L+p}$. In this matrix element, the electron-photon interaction operator is given as a sum of one-particle operators, where $\boldsymbol{\alpha}_{n}$ denotes the vector of the Dirac matrices for the $n$th particle and $\boldsymbol{A}_{L, n}^{p}$ is the usual magnetic $(p=0)$ or electric $(p=$ 1) multipole field [32]. Moreover, in equation (2), the free electron is characterized by the kinetic energy $\epsilon$, the Dirac angular momentum quantum number $\kappa= \pm(j+1 / 2)$ for $l=j \pm 1 / 2$ and the phase shift $\Delta_{\kappa}$.

Equation (1) displays the general form of the statistical tensors $\rho_{k 0}$ for the $\left|\alpha_{f} J_{f}\right\rangle$ excited ionic state produced by electron recombination into many-electron ion. If the capture occurs into an initially bare ion, we have $J_{i}=0$ and $\left(\alpha_{f} J_{f}\right)=\left(n_{b} j_{b}\right)$ in order to designate the bound state of the hydrogen-like ion, and hence the statistical tensors simplify to:

$$
\begin{gathered}
\rho_{k 0}\left(n_{b} j_{b}\right)=32 \pi^{3} \sum_{L p} \sum_{\kappa \kappa^{\prime}}\left[l, l^{\prime}, j, j^{\prime}\right]^{1 / 2}(-1)^{L+j_{b}-1 / 2} \\
\times\left\langle l 0 l^{\prime} 0 \mid k 0\right\rangle\left\{\begin{array}{ccc}
j & j^{\prime} & k \\
l^{\prime} & l & 1 / 2
\end{array}\right\}\left\{\begin{array}{ccc}
j & j^{\prime} & k \\
j_{b} & j_{b} & L
\end{array}\right\} \\
\times\left\langle\epsilon l j\left\|H_{\gamma}(p L)\right\| n_{b} j_{b}\right\rangle\left\langle\epsilon l^{\prime} j^{\prime}\left\|H_{\gamma}(p L)\right\| n_{b} j_{b}\right\rangle^{*},
\end{gathered}
$$

as shown and calculated before at various places in the literature [28,33].

In the next sections, we make use of the independent particle model (IPM) in order to express the statistical tensors of the many-electron (1) ions in terms of their one-electron analogs (3). Before starting the IPM analysis, however, we shall briefly recall that for practical computations, the tensors $\rho_{k 0}$ are usually renormalized with respect to the zero-rank tensor [2-4]:

$$
\mathcal{A}_{k 0}\left(\alpha_{f} J_{f}\right)=\frac{\rho_{k 0}\left(\alpha_{f} J_{f}\right)}{\rho_{00}\left(\alpha_{f} J_{f}\right)} .
$$

These renormalized tensors (or alignment parameters) are independent of the particular normalization of the ion density matrix and can be directly related to the (partial) cross sections $\sigma_{\left|\alpha_{f} J_{f} M_{f}\right\rangle}$ for the population of the different ionic sublevels $\left|\alpha_{f} J_{f} M_{f}\right\rangle$. For the case of the capture of an unpolarized electron into the bound state of the unpolarized ion, the alignment parameters (4) are non-zero only if $k$ is even and $k \leq 2 J_{f}$. This immediately implies that while the magnetic sublevel population of the level with $J_{f}=1$ is described by a single parameter $\mathcal{A}_{20}\left(\alpha_{f} J_{f}=1\right)$, two parameters $\mathcal{A}_{20}\left(\alpha_{f} J_{f}=2\right)$ and $\mathcal{A}_{40}\left(\alpha_{f} J_{f}=2\right)$ are required for $J_{f}=2$.

\subsection{Alignment parameters within the independent particle model}

As seen from expressions (1) and (4), in order to explore the alignment parameters of many-electron heavy ions, we first need to evaluate the (reduced) matrix element $\left\langle\left(\alpha_{i} J_{i}, \epsilon l j\right) J\left\|H_{\gamma}(p L)\right\| \alpha_{f} J_{f}\right\rangle$. Two types of manyelectron states appear on the left-hand side and the righthand side of the matrix element: while initially we have a scattering state with one electron in the continuum (bra), the final state of the ion is given by an ordinary bound state (ket). In our recent works [29,30], these states have been generated within the framework of the multiconfiguration Dirac-Fock (MCDF) method which accounts for the electron-electron interaction effects. Since for the high- $Z$ ions such effects are usually small, we can make use of the IPM to evaluate the free-bound transition amplitude. Within the IPM, the many-electron wave functions are approximated by means of Slater determinants which are built from hydrogenic orbitals in the present work. For this choice of the many-electron function, all the matrix elements can be easily decomposed into the corresponding single-electron amplitudes. For a two-electron system, for example, this decomposition reads as $[34,35]$ :

$$
\begin{aligned}
\left\langle\left(\alpha_{i} J_{i}, \epsilon l j\right) J\left\|H_{\gamma}(p L)\right\| \alpha_{f} J_{f}\right\rangle=(-1)^{j_{b}-J+L+j_{0}} \\
\quad \times\left\{\begin{array}{ccc}
j_{0} & j_{b} & J_{f} \\
L & J & j
\end{array}\right\}\left[J_{f}, J\right]^{1 / 2}\left\langle\epsilon l j\left\|H_{\gamma}(p L)\right\| n_{b} j_{b}\right\rangle .
\end{aligned}
$$

In this expression, $\left|\left(\alpha_{i} J_{i}, \epsilon l j\right) J\right\rangle \equiv\left|\left(n_{0} j_{0}, \epsilon l j\right) J\right\rangle$ and $\left|\alpha_{f} J_{f}\right\rangle \equiv\left|\left(n_{0} j_{0}, n_{b} j_{b}\right) J_{f}\right\rangle$ are the initial (scattering) and the final (bound) states, correspondingly. Moreover, we assume that the initially bound electron in the state $\left|n_{0} j_{0}\right\rangle$ stays passive in the capture process.

Even though the relation (5) has been derived for the electron recombination into initially hydrogen-like ions $\left|\alpha_{i} J_{i}\right\rangle \equiv\left|n_{0} j_{0}\right\rangle$, it can certainly be applied for an arbitrary system with a single valence electron outside of closed shell. In Section 3, for example, we apply equation (5) to study the alignment following the electron capture into the $1 s^{2} 2 s 3 d_{3 / 2} J_{f}=1,2$ states of (initially) lithium-like heavy ions.

The great advantage of the (many-electron) reduced matrix element (5) is that it helps to describe all the properties of the electron capture by many-electron ions in terms of their one-electron analogs. For instance, substituting expression (5) into equation (1) and by using the fact that the zero-rank statistical tensor is equal - up to some geometrical factor - to the total cross section $[2,32]$ :

$$
\rho_{k 0}\left(\alpha_{f} J_{f}\right)=\frac{1}{\sqrt{2 J_{f}+1}} \sigma_{\left|\alpha_{f} J_{f}\right\rangle},
$$

we obtain the simple relation between the cross sections for the radiative electron capture into the excited states $\left|\left(n_{0} j_{0}, n_{b} j_{b}\right) J_{f}\right\rangle$ of the helium-like and the excited states $\left|n_{b} l_{b} j_{b}\right\rangle$ of hydrogen-like ions, respectively:

$$
\sigma_{\left|\alpha_{f} J_{f}\right\rangle}=\frac{2 J_{f}+1}{\left(2 j_{0}+1\right)\left(2 j_{b}+1\right)} \sigma_{\left|n_{b} j_{b}\right\rangle} .
$$

From this relation, one can immediately derive the wellknown statistical ratio:

$$
\frac{\sigma_{\left|\alpha_{f} J_{f}^{(1)}\right\rangle}}{\sigma\left|\alpha_{f} J_{f}^{(2)}\right\rangle}=\frac{2 J_{f}^{(1)}+1}{2 J_{f}^{(2)}+1}
$$


for the electron capture cross sections and, hence, the populations of the fine-structure levels $\left|\left(n_{0} j_{0}, n_{b} j_{b}\right) J_{f}^{(1)}\right\rangle$ and $\left|\left(n_{0} j_{0}, n_{b} j_{b}\right) J_{f}^{(2)}\right\rangle$.

Similar to the total cross sections, equation (5) helps also to express the alignment parameters $\mathcal{A}_{k 0}\left(\alpha_{f} J_{f}\right)$ of the helium-like ions in terms of the reduced tensors $\mathcal{A}_{k 0}\left(n_{b} j_{b}\right)$ that describe the magnetic sublevel population of the of hydrogen-like ions:

$$
\begin{aligned}
\mathcal{A}_{k 0}\left(\alpha_{f} J_{f}\right)=\left[j_{b}, J_{f}\right]^{1 / 2}(-1)^{J_{f}+j_{b}+j_{0}} & \\
& \times\left\{\begin{array}{lll}
J_{f} & J_{f} & k \\
j_{b} & j_{b} & j_{0}
\end{array}\right\} \mathcal{A}_{k 0}\left(n_{b} j_{b}\right) .
\end{aligned}
$$

As seen from this equation, the alignment calculations for the single excited states of the two-electron (or even many-electron) ions can be traced back always to the oneelectron parameters $\mathcal{A}_{k 0}\left(n_{b} j_{b}\right)$. Since these parameters are tabulated in reference [28] for medium- and high- $Z$ ions over a wide range of projectile energies, equation (9) provides a fast access to the alignment of few-electron ions as required often for the analysis of electron-capture experiments $[19,26]$. Note however, that equation (9) was obtained by omitting the electron-electron interaction effects and, hence, is an approximation. In Section 3.1, therefore, we shall perform extensive numerical check of equation (9) in order to find the limits of its applicability.

\subsection{Subsequent radiative decay}

As discussed above for the electron capture into excited ionic states $\left|\alpha_{f} J_{f}\right\rangle$, the alignment parameters $\mathcal{A}_{k 0}\left(\alpha_{f} J_{f}\right)$ are closely related also to the angular and polarization properties of the subsequent decay radiation. For example, the angular distribution of the photons which are emitted in course of the $\left|\alpha_{f} J_{f}\right\rangle \rightarrow \gamma+\left|\alpha_{0} J_{0}\right\rangle$ decay is given by

$$
\begin{aligned}
W(\theta)=\frac{\sigma_{0}^{\mathrm{dec}}}{4 \pi}\left(1+\sum_{k=2,4, . .}\right. & f_{k}^{\mathrm{dec}}\left(\alpha_{f} J_{f}, \alpha_{0} J_{0}\right) \\
& \left.\times \mathcal{A}_{k 0}\left(\alpha_{f} J_{f}\right) P_{k}(\cos \theta)\right),
\end{aligned}
$$

where $\sigma_{0}^{\text {dec }}$ is the total decay rate and $\theta$ denotes the angle of the photons with respect to the collision direction (quantization axis). One may see from this equation that, apart from the reduced statistical tensors, the photon emission pattern also depends on the so-called anisotropy parameters $f_{k}^{\mathrm{dec}}\left(\alpha_{f} J_{f}, \alpha_{0} J_{0}\right)$. The general expression for these parameters, which accounts for the possible multipole mixing effects, is given in references $[29,36]$. Since in the present work, we shall focus onto the formation and decay of helium-like and beryllium-like ions, we consider the radiative transitions into the $J_{0}=0$ ground state, for which the $f_{k}^{\text {dec }}\left(\alpha_{f} J_{f}, \alpha_{0} J_{0}=0\right)$ then reads:

$$
\begin{aligned}
f_{k}^{\text {dec }}\left(\alpha_{f} J_{f}, \alpha_{0} J_{0}=0\right)=(-1)^{1-} & J_{f} \sqrt{2 J_{f}+1} \\
& \times\left\langle J_{f} 1 J_{f}-1 \mid k 0\right\rangle .
\end{aligned}
$$

Based in equations (10) and (11), we may immediately obtain the angular distributions of the $J_{f}=1 \rightarrow J_{0}=0$ :

$$
W(\theta)=\frac{\sigma_{0}^{\mathrm{dec}}}{4 \pi}\left(1+\frac{1}{\sqrt{2}} \mathcal{A}_{20}\left(\alpha_{f} J_{f}=1\right) P_{2}(\cos \theta)\right),
$$

and $J_{f}=2 \rightarrow J_{0}=0$ transition:

$$
W(\theta)=\frac{\sigma_{0}^{\mathrm{dec}}}{4 \pi}\left(1-\sqrt{\frac{5}{14}} \mathcal{A}_{20}\left(\alpha_{f} J_{f}=2\right) P_{2}(\cos \theta)\right) .
$$

As seen from these expressions, the knowledge of the (many-electron) alignment parameters $\mathcal{A}_{20}\left(\alpha_{f} J_{f}=1,2\right)$ is required for studying of the angular distribution of the $J_{f}=1,2 \rightarrow J_{0}=0$ X-ray lines. Such a study, however, can be simplified considerably if equation (9), which was obtained within the effective one electron model, is utilized for the alignment calculations. An example of such IPM calculation, applied to the $\mathrm{K} \alpha_{1}$ decay in helium-like ions, will be discussed below in Section 3.2.

In Section 3.2, furthermore, we shall see that apart from the alignment parameters, the decay rates $\sigma_{0}^{\text {dec }}$ of the (helium-like) excited states have to be calculated for the proper analysis of the $\mathrm{K} \alpha_{1}$ emission pattern. For high- $Z$ domain, these rates can also be estimated from the independent particle model. That is, by making decomposition of the two-electron transition amplitudes in terms of the corresponding single-electron matrix elements (similar to that leading to Eq. (5)), we may find the relation

$$
\begin{aligned}
\sigma_{0}^{\operatorname{dec}}\left(\alpha_{f} J_{f}, \alpha_{0} J_{0}\right) & =\left(2 J_{0}+1\right)\left(2 j_{b}+1\right)\left\{\begin{array}{ccc}
j_{0}^{\prime} & j_{0} & J_{0} \\
J_{f} & L & j_{b}
\end{array}\right\}^{2} \\
& \times\left(1+\delta_{n_{0}^{\prime} n_{0}} \delta_{j_{0}^{\prime} j_{0}}\right) \sigma_{0}^{\operatorname{dec}}\left(n_{b} j_{b}, n_{0}^{\prime} j_{0}^{\prime}\right)
\end{aligned}
$$

between the rates for emission of a photon with angular momentum $L$ in the $\left|\left(n_{0} j_{0}, n_{b} j_{b}\right) J_{f}\right\rangle \rightarrow\left|\left(n_{0} j_{0}, n_{0}^{\prime} j_{0}^{\prime}\right) J_{0}\right\rangle$ and $\left|n_{b} j_{b}\right\rangle \rightarrow\left|n_{0}^{\prime} j_{0}^{\prime}\right\rangle$ decay of helium-like and hydrogenlike ions, correspondingly.

\section{Results and discussion}

\subsection{Alignment of the helium- and beryllium-like heavy ions}

Motivated by recent experimental studies [19,26], a number of theoretical works were done to calculate the alignment of helium-like and beryllium-like heavy ions produced by electron capture into excited ionic states $[29,30]$. Until now, most of the calculations have been performed within the framework of the multiconfiguration DiracFock approach which allows a systematic account for the interelectronic interaction effects. This approach is implemented, for example, in the RATIP program [37], that now facilitates the computation of the REC total and angle-differential cross sections as well as the alignment parameters. By employing the MCDF method, significant alignment was found for the $1 s 2 p_{3 / 2} J_{f}=1,2$ excited states of helium-like medium- $Z$ and high- $Z$ ions. 


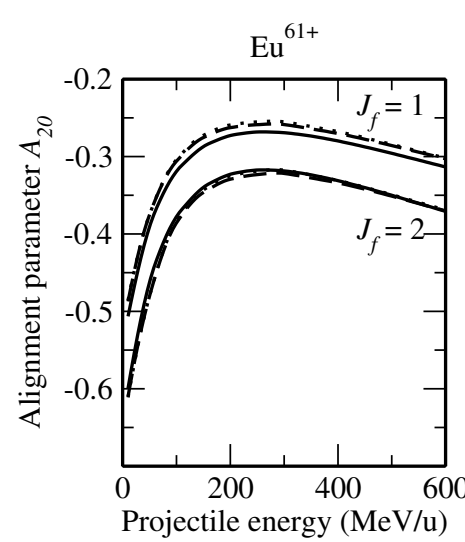

For example, as seen from Figure 1, the reduced tensors $\mathcal{A}_{20}\left(1 s 2 p_{3 / 2} J_{f}=1\right)$ and $\mathcal{A}_{20}\left(1 s 2 p_{3 / 2} J_{f}=2\right)$ of the uranium ions $\mathrm{U}^{90+}$ are as large as -0.56 and -0.66 for $J_{f}=1$ and $J_{f}=2$ at projectile energy $T_{p}=10 \mathrm{MeV} / \mathrm{u}$ and slightly decrease to -0.36 and -0.43 for higher energies. Although for the lower nuclear charges such an alignment is reduced by about $10 \%$ to $20 \%$, it is still significant enough to modify the linear polarization and angular distribution of the subsequent decay photons.

After this short discussion of the previous MCDF calculations, we now like to re-investigate the alignment of the $1 s 2 p_{3 / 2} J_{f}=1,2$ states of helium-like ions by means of our - simple but efficient - independent particle model. To this end, let us start from the parameters $\mathcal{A}_{20}\left(2 p_{3 / 2}\right)$ for the electron capture into the $2 p_{3 / 2}$ state of (initially) bare ions. The evaluation of these one-electron parameters within the framework of the relativistic Dirac theory has been discussed previously at a number of places $[28,33]$. Below, we calculate the statistical tensors $\mathcal{A}_{20}\left(2 p_{3 / 2}\right)$ by using the DIRAC program [38]. Since these (one-particle) tensors are used later also to explore the alignment of the helium-like ions, the screening of the nucleus by the core electron should be taken into account right from the beginning by a proper choice of the effective charge $Z_{\text {eff }}$. In the calculations below, this effective charge of the hydrogenic wavefunction was chosen to adjust the "true" transition energy for the recombination of a free electron into $1 s_{1 / 2} 2 p_{3 / 2}$ excited states of the helium-like ions. Because of the energy conservation, which relates the transition energy to the kinetic energy of a free (quasi-free) electron as well as to the total energies of the ion just before and after the recombination, the problem of determining the effective charge $Z_{\text {eff }}$ can be traced back to the binding energy of the $2 p_{3 / 2}$ electron in the helium-like ions. For the $1 s_{1 / 2} 2 p_{3 / 2}$ states of the europium, gold and uranium ions these binding energies are estimated from the MCDF calculations as $E_{b, 2 p_{3 / 2}}(\mathrm{Eu})=4.8 \times 10^{2}, E_{b, 2 p_{3 / 2}}(\mathrm{Au})=$ $7.8 \times 10^{2}$ and $E_{b, 2 p_{3 / 2}}(\mathrm{U})=1.1 \times 10^{3}$ a.u. which implies the following choice of the effective charges: $Z_{\text {eff }}(\mathrm{Eu})=$ $62.0, Z_{\text {eff }}(\mathrm{Au})=78.1$ and $Z_{\text {eff }}(\mathrm{U})=91.1$, respectively. By making use of these effective charges we are able to evaluate the statistical tensors $\mathcal{A}_{20}\left(2 p_{3 / 2}\right)$ and substitute them into equation (9) in order to obtain the alignment $\mathcal{A}_{20}\left(1 s 2 p_{3 / 2} J_{f}=1,2\right)$ of the residual helium-like ions.

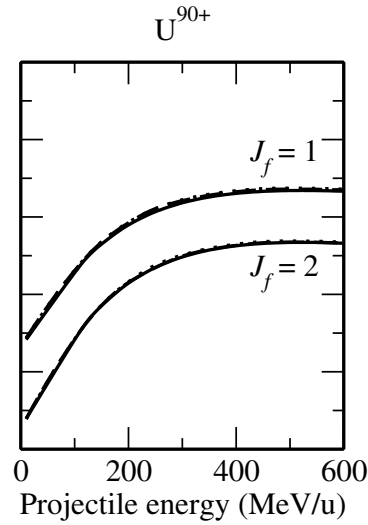

Fig. 1. Alignment parameters $\mathcal{A}_{20}$ of the $1 s 2 p_{3 / 2} J_{f}=1,2$ states of helium-like europium, gold and uranium following the REC into the initially hydrogen-like projectiles. Results from an independent particle model (solid line) are compared with those from MCDF calculations in the Coulomb (dotted line) and in the Babushkin (dashed line) gauge.

Results from these IPM calculations are compared in Figure 1 with the data from the MCDF approach in Coulomb (velocity) and Babushkin (length) gauge. As seen from this figure, good agreement between the oneand many-electron computations is found for the electron recombination into the $J_{f}=1,2$ states of (initially) hydrogen-like gold $\mathrm{Au}^{78+}$ and uranium $\mathrm{U}^{91+}$ ions with energies in the range $10 \leq T_{p} \leq 600 \mathrm{MeV} / \mathrm{u}$. Such an agreement was certainly expected since for energetic, high- $Z$ projectiles the interelectronic effects can be assumed to be negligible. In contrast, if the nuclear charge $Z$ is reduced, the repulsion among the bound-state and free electrons shall stronger affect the properties of the REC. For the recombination into europium ions $\mathrm{Eu}^{61+}$, for example, the many-electron effects lead to a slight decrease of the alignment parameter $\mathcal{A}_{20}\left(1 s 2 p_{3 / 2} J_{f}=1\right)$ when compared with IPM calculations. However, this reduction (of alignment) of $\leq 3 \%$ is well below of the accuracy which can be achieved in current experiments and, hence, we can conclude that the use of the effective one-particle approach is still well justified, at least for medium- $Z$ heliumlike ions.

Our analysis of the effective one-electron approach would be, of course, incomplete if we consider only the capture into the lower-lying excited ionic states. In Figure 2, therefore, we display the parameters $\mathcal{A}_{20}\left(1 s 3 p_{3 / 2} J_{f}=\right.$ $1,2)$ of the $1 s 3 p_{3 / 2} J_{f}=1,2$ states of the helium-like, heavy ions. Similar as before, calculations have been performed within the IPM and the MCDF approaches. For the IPM model calculations, we have chosen again the effective charges $Z_{\text {eff }}(\mathrm{Eu})=62.0, Z_{\text {eff }}(\mathrm{Au})=78.0$ and $Z_{\text {eff }}(\mathrm{U})=91.1$ so as to reproduce the correct value of the $3 p_{3 / 2}$ binding energy in the helium-like ions. As seen from Figure 2, both - one-electron and many-electron approximations yield basically identical results for gold and uranium projectiles but start to differ as the nuclear charge decreases. Moreover, by comparing Figures 1 and 2, one can see a very similar energy behavior of the alignment parameters of the $1 s 2 p_{3 / 2} J_{f}=1,2$ and $1 s 3 p_{3 / 2} J_{f}=$ 1,2 states. This can be well understood within the independent particle model in which the statistical tensors $\mathcal{A}_{20}\left(1 s 2 p_{3 / 2} J_{f}=1,2\right)$ and $\mathcal{A}_{20}\left(1 s 3 p_{3 / 2} J_{f}=1,2\right)$ are just proportional to the $\mathcal{A}_{20}\left(2 p_{3 / 2}\right)$ and $\mathcal{A}_{20}\left(3 p_{3 / 2}\right)$, correspondingly (cf. Eq. (9)). In turn, both one-electron 

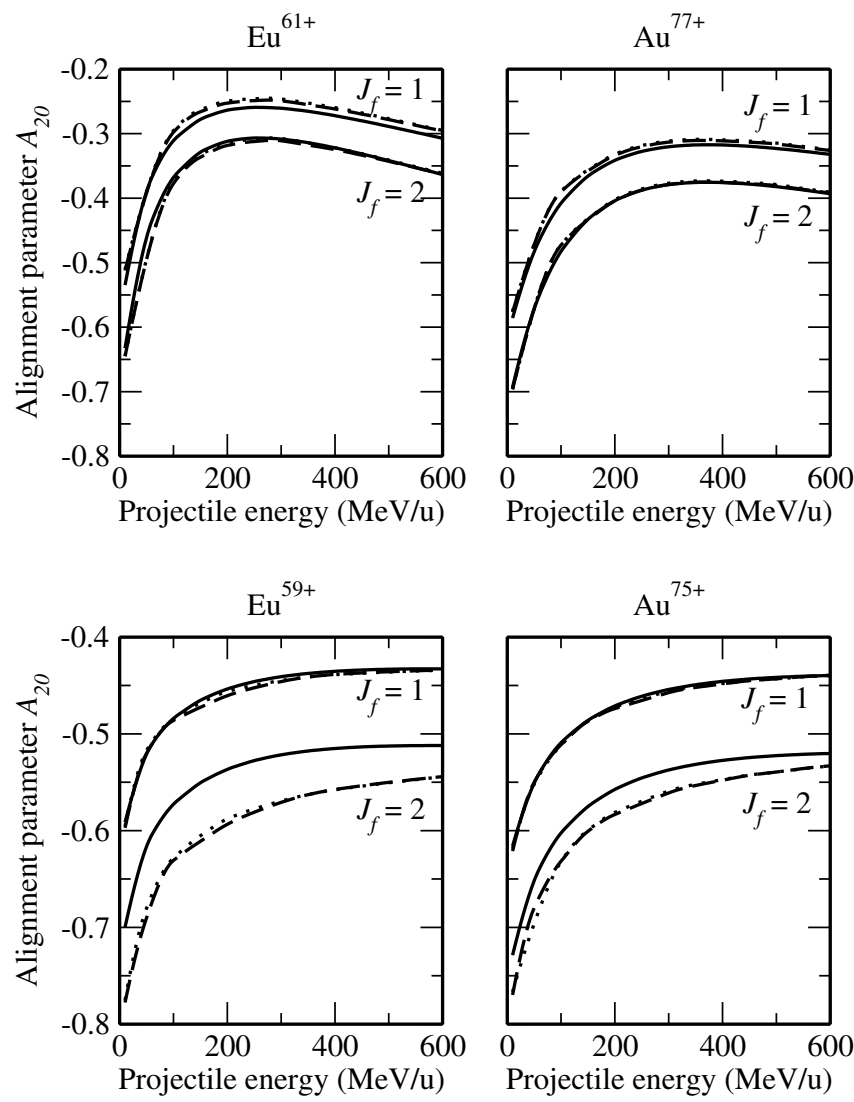

parameters describe the alignment of the states with the same symmetry and just differ in the radial integrals for the $2 p_{3 / 2}$ as compared to the $3 p_{3 / 2}$ state [28].

As seen from the Figures 1 and 2, for the capture into the $1 s 2 p_{3 / 2} J_{f}=1,2$ and $1 s 3 p_{3 / 2} J_{f}=1,2$ states of helium-like ions, we have calculated so far only the alignment parameter $\mathcal{A}_{20}$ of the second rank. Apart from this parameter, the magnetic sublevel population of the levels with $J_{f}=2$ also entails the reduced statistical tensor $\mathcal{A}_{40}$. From our previous MCDF calculations [29], however, we found that the fourth-rank tensors do not exceed a value of $10^{-4}$ for capture into the both $1 s 2 p_{3 / 2}$ and $1 s 3 p_{3 / 2}$ states and for the projectile energies in the range $10 \leq T_{p} \leq$ $600 \mathrm{MeV} / \mathrm{u}$. Such an (almost) vanishing alignment again verifies the predictions $\mathcal{A}_{40}\left(1 s 2 p_{3 / 2} J_{f}=2\right)=0$ and $\mathcal{A}_{40}\left(1 s 3 p_{3 / 2} J_{f}=2\right)=0$ from the IPM which arise from the fact that the statistical tensors of the fourth rank are identically zero for all $n p_{3 / 2}$ one-electron states $[28,33]$.

Up to the present, we have discussed the alignment of helium-like, heavy ions following electron recombination. For these ions, good agreement was found between the results of a simple one-particle model and of the MCDF approach. In order to get a more complete picture of the electron-electron contributions to the magnetic sublevel population of the excited ionic states and, hence, to verify the applicability of the IPM, we shall consider now the capture into initially lithium-like ions. For the $M$ REC by such ions, significant many-particle effects have been reported recently which influence both the angular

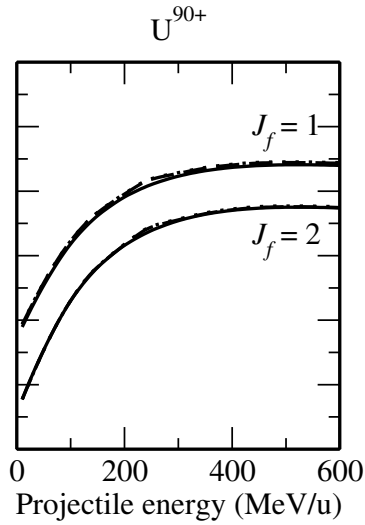

Fig. 2. Alignment parameters $\mathcal{A}_{20}$ of the $1 s 3 p_{3 / 2} J_{f}=1,2$ states of helium-like europium, gold and uranium following the REC into the initially hydrogen-like projectiles. Results from an independent particle model (solid line) are compared with those from MCDF calculations in the Coulomb (dotted line) and in the Babushkin (dashed line) gauge.

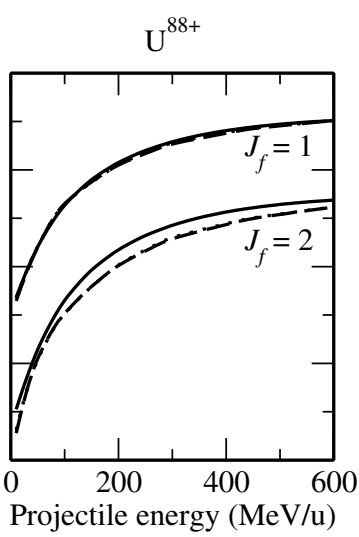

Fig. 3. Alignment parameters $\mathcal{A}_{20}$ of the $1 s^{2} 2 s 3 d_{3 / 2} J_{f}=1,2$ states of beryllium-like europium, gold and uranium following the REC into the initially lithium-like projectiles. Results from an independent particle model (solid line) are compared with those from MCDF calculations in the Coulomb (dotted line) and in the Babushkin (dashed line) gauge.

distribution of the recombination photons and the spin states of the residual ion $[29,32]$. In Figure 3, therefore, we display the results of the IPM and MCDF calculations for the alignment $\mathcal{A}_{20}$ of the $1 s^{2} 2 s 3 d_{3 / 2} J_{f}=1,2$ states of beryllium-like europium, gold and uranium ions. As seen from this figure, while good agreement between one- and many-particle computations can be observed for the capture into the $J_{f}=1$ level, some deviation appears for $J_{f}=2$. In particular, the independent particle model systematically underestimates the (absolute value of) alignment $\mathcal{A}_{20}\left(2 s 3 d_{3 / 2} J_{f}=2\right)$; this effect becomes more significant as the nuclear charge decreases. Such a deviation from MCDF results arises mainly due to the mixture of the $1 s^{2} 2 s 3 d_{3 / 2} J_{f}=2$ level with other levels having a stronger alignment. From our calculations, for instance, we found a (relatively) large admixture of the $1 s^{2} 2 s 3 d_{5 / 2} J_{f}=2$ level for which a large positive parameter $\mathcal{A}_{20}\left(2 s 3 d_{5 / 2} J_{f}=2\right)$ has been predicted before [29]. This admixture increases from $0.5 \%$ for $\mathrm{U}^{88+}$ to almost $5 \%$ for lower nuclear charges as expected from the $1 / Z$ scaling law for the electromagnetic interaction between the electrons. Besides the enhancement of the alignment parameter $\mathcal{A}_{20}$, the configuration interaction effects also result in the non-zero fourth rank parameter $\mathcal{A}_{40}$ which otherwise identically vanishes within the independent particle model. As seen from Figure 4, this parameter is, however, rather small for the electron recombination into the uranium projectiles but significantly increases for lighter elements. For the beryllium-like europium ions 


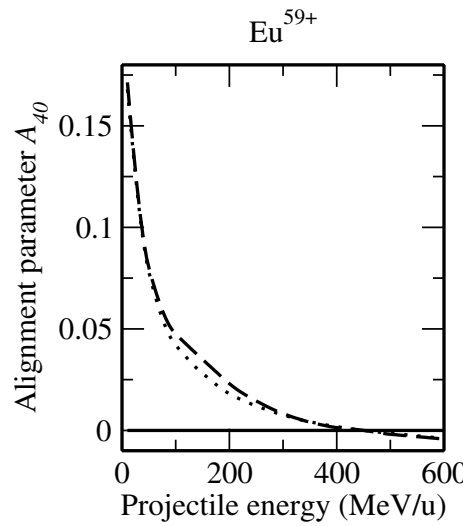

with projectile energy $T_{p}=10 \mathrm{MeV} / \mathrm{u}$, for example, the MCDF calculations predict that the alignment parameter $\mathcal{A}_{40}$ is almost 0.17 and, hence, could possibly be detected using presently available X-ray detectors.

\subsection{Isotropy of the $\mathrm{K} \alpha_{1}$ radiation from the helium-like uranium ions}

As seen from the results presented in Figures 1-4, the independent particle model provides a reasonable estimate of the alignment parameters of excited states of high- $Z$, helium-like and beryllium-like ions following REC. This model, therefore, can be also applied for the analysis of the angular distribution and polarization of the subsequent decay radiation; these properties are directly related to the reduced tensors $\mathcal{A}_{k 0}$ (cf. Eq. (10)). In order to illustrate such an analysis, we now make use of equation (9) for studying the angular distribution of the $\mathrm{K} \alpha_{1}\left(1 s 2 p_{3 / 2} J_{f}=1,2 \rightarrow 1 s^{2} J_{0}=0\right)$ radiation from helium-like uranium ions $\mathrm{U}^{90+}$ produced by electron recombination. Experiments concerning this radiative decay have recently been performed at the GSI storage ring, and the angular distributions observed have been found to be qualitatively different from those of the Lyman$\alpha_{1}\left(2 p_{3 / 2} \rightarrow 1 s\right)$ decay in the hydrogen-like ions $[19,26]$. Namely, while the Lyman- $\alpha_{1}$ radiation exhibited a strong angular dependence, the $\mathrm{K} \alpha_{1}$ decay gave rise to an almost isotropic emission pattern. Recently, it has been found that such an isotropy results from the mutual cancellation of the angular distributions of the - strongly anisotropic $-J_{f}=1 \rightarrow J_{0}=0$ electric dipole and $J_{f}=2 \rightarrow J_{0}=0$ magnetic quadrupole transitions, both of which contribute to the $\mathrm{K} \alpha_{1}$ radiation [30,31]. Mathematically, this cancellation implies a vanishing effective anisotropy parameter $\beta_{2}^{\text {eff }}$ which governs the shape of the $\mathrm{K} \alpha_{1}$ angular distribution

$$
W(\theta)_{\mathrm{K} \alpha_{1}} \sim 1+\beta_{2}^{\mathrm{eff}}\left(\mathrm{K} \alpha_{1}\right) P_{2}(\cos \theta) .
$$

By using equations (12) and (13), a general expression for the anisotropy parameter $\beta_{2}^{\text {eff }}$ can be derived:

$$
\begin{aligned}
\beta_{2}^{\mathrm{eff}}\left(\mathrm{K} \alpha_{1}\right)= & N_{1} \mathcal{A}_{20}\left(1 s 2 p_{3 / 2} J_{f}=1\right) \frac{1}{\sqrt{2}} \\
& -N_{2} \mathcal{A}_{20}\left(1 s 2 p_{3 / 2} J_{f}=2\right) \sqrt{\frac{5}{14}},
\end{aligned}
$$

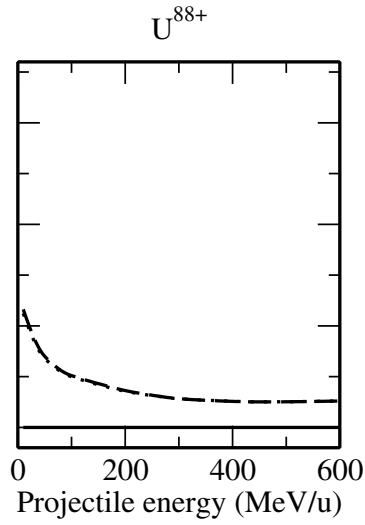

Fig. 4. Alignment parameters $\mathcal{A}_{40}$ of the $1 s^{2} 2 s 3 d_{3 / 2} J_{f}=2$ state of beryllium-like europium, gold and uranium following the REC into the initially lithium-like projectiles. Results from an independent particle model (solid line) are compared with those from MCDF calculations in the Coulomb (dotted line) and in the Babushkin (dashed line) gauge. where $\mathcal{A}_{20}\left(1 s 2 p_{3 / 2} J_{f}=1,2\right)$ are the alignment parameters of the $1 s 2 p_{3 / 2} J_{f}=1$ and $1 s 2 p_{3 / 2} J_{f}=2$ states, and $N_{1,2}$ are the weights which describe the contribution of the individual $J_{f}=1 \rightarrow J_{0}=0$ and $J_{f}=2 \rightarrow J_{0}=0$ transitions to the overall $\mathrm{K} \alpha_{1}$. These (relative) weights, are assumed to fulfill the normalization condition $N_{1}+N_{2}=1$.

Detailed calculations based on the MCDF approach have been performed for the anisotropy parameter (16) and proved almost isotropic behavior of the $\mathrm{K} \alpha_{1}$ angular distribution as it was observed in the experiments. Despite a good numerical estimation for the results of the measurements, equation (16) can hardly be applied for the interpretation of experimental data since it depends on too many independent parameters. Below, therefore, we like to simplify this expression by making use of the IPM. That is, we first employ equation (9) to express the alignment parameters $\mathcal{A}_{20}\left(1 s 2 p_{3 / 2} J_{f}=1,2\right)$ of the $1 s 2 p_{3 / 2} J_{f}=1,2$ helium states in terms of the one-electron reduced tensors $\mathcal{A}_{20}\left(2 p_{3 / 2}\right)$ :

$$
\mathcal{A}_{20}\left(1 s 2 p_{3 / 2} J_{f}=1\right)=\frac{1}{\sqrt{2}} \mathcal{A}_{20}\left(2 p_{3 / 2}\right),
$$

and

$$
\mathcal{A}_{20}\left(1 s 2 p_{3 / 2} J_{f}=2\right)=\sqrt{\frac{7}{10}} \mathcal{A}_{20}\left(2 p_{3 / 2}\right) .
$$

By inserting now these two alignment parameters into equation (16), we obtain the effective anisotropy parameter $\beta_{2}^{\text {eff }}$ in the simple form:

$$
\beta_{2}^{\mathrm{eff}}\left(\mathrm{K} \alpha_{1}\right)=\frac{1}{2} \mathcal{A}_{20}\left(2 p_{3 / 2}\right)\left(N_{1}-N_{2}\right),
$$

which allows immediate interpretation of experimental data. As seen from this equation, the parameter $\beta_{2}^{\text {eff }}\left(\mathrm{K} \alpha_{1}\right)$, as derived within the IPM, appears to be very similar to the anisotropy parameter $\beta_{2}\left(\operatorname{Ly} \alpha_{1}\right)=$ $\mathcal{A}_{20}\left(2 p_{3 / 2}\right) f(E 1, M 2) / 2$ of the Lyman- $\alpha_{1}$ radiation from one-electron ions $[27,33]$. The only difference is that in place of the structure function $f(E 1, M 2)$ which describes the multipole mixing between the electric dipole and magnetic quadrupole components of the Lyman- $\alpha_{1}$ transition, one obtains in equation (19) the difference between the statistical weights $N_{1}$ and $N_{2}$. Such a difference implies two phenomenologically important constraints: (i) since 
$f(E 1, M 2)>1$ for all nuclear charges $Z$, the anisotropy of the $\mathrm{K} \alpha_{1}$ radiation is always weaker than one in the Lyman- $\alpha_{1}$ case, (ii) an almost isotropic behavior of the $\mathrm{K} \alpha_{1}$ angular distribution can be obtained if one assumes equal contributions of its fine-structure $J_{f}=1 \rightarrow J_{0}=0$ and $J_{f}=2 \rightarrow J_{0}=0$ components: $N_{1} \approx N_{2}$.

As seen from equation (19) and the discussion above, in order to understand the (observed $[19,26]$ ) isotropy of the $\mathrm{K} \alpha_{1}$ radiation from the helium-like, uranium ions we have to consider closer the weight parameters $N_{1,2}$. These parameters shall account not only for the relative population of the $J_{f}=1,2$ levels following REC but also for their decay branching fractions. Both, the relative populations and the branching ratios can be easily estimated within the effective one-particle approach. In particular, by making use of equation (8) we find the statistical ratio $\left(N_{1} / N_{2}\right)_{R E C}=3 / 5$ of the level populations as arises from the electron capture. In order to analyze the possible depopulation mechanisms of the helium-like systems we shall employ equation (14). For example, this equation helps to obtain the ratio of the transition probabilities for the $1 s 2 p_{3 / 2} J_{f}=2 \rightarrow 1 s 2 s J_{0}=1$ (electric dipole) and $1 s 2 p_{3 / 2} J_{f}=2 \rightarrow 1 s^{2} J_{0}=0$ (magnetic quadrupole) competitive decay channels in the helium-like uranium $\mathrm{U}^{90+}$ :

$$
\begin{aligned}
\frac{\sigma_{0}^{\operatorname{dec}}\left(J_{f}=2 \rightarrow\right.}{\sigma_{0}^{\operatorname{dec}}\left(J_{f}=2 \rightarrow\right.} & \left.J_{0}=1(E 1)\right) \\
& \frac{5}{4} \frac{\sigma_{0}^{\operatorname{dec}}\left(2 p_{3 / 2} \rightarrow 2 s_{1 / 2}(E 1)\right)}{\sigma_{0}^{\operatorname{dec}}\left(2 p_{3 / 2} \rightarrow 1 s_{1 / 2}(M 2)\right)} \approx 0.41,
\end{aligned}
$$

where the one-electron decay rates have been calculated within the Dirac's relativistic framework [38] and by using the effective charges $Z_{\text {eff }}^{E 1}=91.7$ and $Z_{\text {eff }}^{M 2}=91.2$ as estimated from the "true" transition energies. From equation (20), one may see that a strong $1 s 2 p_{3 / 2} J_{f}=2 \rightarrow$ $1 s 2 s J_{0}=1$ transition leads to the fact that only about $70 \%$ of the population of the triplet $1 s 2 p_{3 / 2} J_{f}=2$ state contributes to the $\mathrm{K} \alpha_{1}$ decay. When this branching ratio is taken into account, we immediately find that the weights of the $J_{f}=1 \rightarrow J_{0}=0$ and $J_{f}=2 \rightarrow J_{0}=0$ finestructure components in the $\mathrm{K} \alpha_{1}$ transition are almost equal:

$$
\begin{aligned}
\frac{N_{1}}{N_{2}} & =\left(\frac{N_{1}}{N_{2}}\right)_{R E C}\left(1+\frac{5}{4} \frac{\sigma_{0}^{\operatorname{dec}}\left(2 p_{3 / 2} \rightarrow 2 s_{1 / 2}(E 1)\right)}{\sigma_{0}^{\operatorname{dec}}\left(2 p_{3 / 2} \rightarrow 1 s_{1 / 2}(M 2)\right)}\right) \\
& \approx 0.843
\end{aligned}
$$

as it was expected from the analysis of the experimental data on the basis of equation (19). We like to note here that the simple formula (21), derived by using the IPM, provides a good estimate to the ratio $N_{1} / N_{2} \approx$ 0.855 as could be obtained from the many-electron treatment of the branching fractions by Johnson, Plante and Sapirstein [39].

Apart from the clear qualitative interpretation of the experimental data, equation (19) provides also reasonable quantitative estimate of the anisotropy of the $\mathrm{K} \alpha_{1}$ radiation. Namely, by inserting the (more accurate) ratio

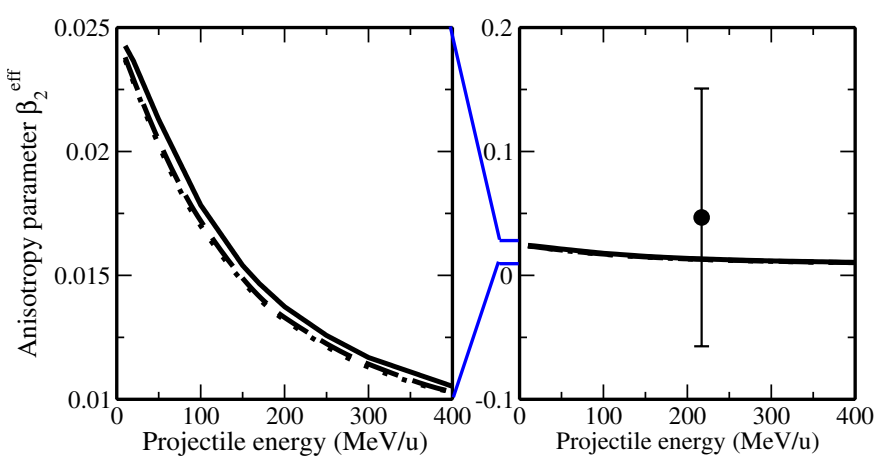

Fig. 5. Anisotropy parameter $\beta_{2}^{\text {eff }}\left(K \alpha_{1}\right)$ of the $K \alpha_{1}$ radiation following REC into excited states of initially hydrogen-like uranium ions. At the right panel results from an independent particle model (solid line) as well as from MCDF calculations in the Coulomb (dotted line) and in the Babushkin (dashed line) gauge are compared with experimental data (solid point). For both IPM and MCDF approaches, theoretical calculations include the feeding transitions from the higher excited states. The left panel is a close-up of the right one, where the range of the ordinate axis in restricted to the interval $0.01 \leq \beta_{2}^{\text {eff }} \leq 0.025$ and the experimental point is not depicted.

$N_{1} / N_{2} \approx 0.855$ into this equation, we may find for the helium-like uranium ions $\mathrm{U}^{90+}$ :

$$
\beta_{2}^{\mathrm{eff}}\left(\mathrm{K} \alpha_{1}\right) \approx-0.039 \mathcal{A}_{20}\left(2 p_{3 / 2}\right)
$$

The computations of the anisotropy $\beta_{2}^{\text {eff }}\left(\mathrm{K} \alpha_{1}\right)$ require, therefore, only the knowledge about the alignment parameter of the $2 p_{3 / 2}$ state of hydrogen-like uranium following REC. Similar to before, this parameter has been calculated by using the computer code DIRAC [38]. In contrast to the results of Section 3.1, however, besides the direct electron capture into the $2 p_{3 / 2}$ state, the cascade feeding from the high-lying levels has also been taken into account. The results of these calculations are presented in Figure 5 as a function of projectile energy $T_{p}$ and are compared with our previous predictions for the $\mathrm{K} \alpha_{1}$ anisotropy based on the MCDF approach (see Ref. [30] for further details). For the energy $T_{p}=217 \mathrm{MeV} / \mathrm{u}$, moreover, we also display the experimental $\beta_{2}^{\text {eff }}\left(\mathrm{K} \alpha_{1}\right)$ value. This value was obtained at the internal target of the ESR storage ring at GSI for initially He-like uranium projectiles colliding with $\mathrm{N}_{2}$ molecules. For a detailed description of the experimental techniques we refer to reference [25] and just mention that the experimental parameter $\beta_{2}^{\text {eff }}$ has been extracted from the fitting of the (measured) emission pattern of the $\mathrm{K} \alpha_{1}$ radiation to equation (15).

As seen from the Figure 5, the deviation between the one- and many-electron calculations is very small and does not exceed $1 \%$ along the whole range of energies. Obviously, this is much smaller than the experimental accuracy displayed and indicates that the independent particle approach (19) can well be applied for the analysis of the experimental data on the characteristic X-ray emission from the heavy, few-electron ions. 


\section{Summary and outlook}

In conclusion, the density matrix approach has been applied for studying the radiative capture of free (or quasifree) electrons into the excited states of open-shell heavy ions. In our theoretical analysis, special emphasis was placed particularly on the magnetic sublevel population of the residual ions which is described by set of the alignment parameters $\mathcal{A}_{k 0}$. General expressions for the alignment parameters have been recalled in terms of the (manyelectron) matrix elements which describe the bound-free electron transition under the simultaneous photon emissions. While, in general, the evaluation of these matrix elements requires the knowledge of the "true" many-particle wavefunctions, in our present work we made use of Dirac hydrogenic spinors and a proper set of Slater determinants to account for the Pauli principle. By making use of the standard reduction of the multi-electron matrix element to the one-particle amplitude, we were able to trace the computation of the (many-electron) alignment parameters $\mathcal{A}_{k 0}$ back to their hydrogenic analogs. In order to check the validity of the IPM, detailed calculations were performed for the alignment of the excited states of the helium- and beryllium-like ions following REC. From the comparison (of the results) of these calculations with the data from the multiconfiguration Dirac-Fock approach, we found that an effective one-particle approximation is sufficient to describe the alignment of the excited states of the high- $Z$, few-electron ions. In particular, the results from the IPM and MCDF approaches deviate not more than $1-3 \%$, i.e. well below the accuracy of the present-day alignment experiments. Therefore, the formulas based on the effective one-particle model can be used for a simple preliminary analysis of the experimental data and enable one to avoid lengthy and often quite sophisticated manyparticle calculations.

Besides providing a simple approach to calculate the alignment of the excited ionic states following REC, the effective one-particle model can also be applied for studying their subsequent radiative decay. This becomes possible due to the fact that the angular and polarization properties of the characteristic X-ray photons are closely related to the alignment parameters. For instance, by making use of the statistical tensors $\mathcal{A}_{k 0}\left(1 s 2 p_{3 / 2} J_{f}=1,2\right)$ as obtained within the IPM, we were able to simplify the anisotropy parameter of the $\mathrm{K} \alpha_{1}\left(1 s 2 p_{3 / 2} J_{f}=1,2 \rightarrow\right.$ $\left.1 s^{2} J_{0}=0\right)$ radiation from the helium-like heavy ions produced by electron capture. This characteristic X-ray emission has been observed recently at the GSI storage ring in Darmstadt $[19,26]$ giving rise to a surprisingly isotropic angular distribution. With the help of the simplified anisotropy parameter, we found that the observed isotropy results from the mutual cancellation of the angular distributions of the (strongly anisotropic) $J_{f}=1 \rightarrow$ $J_{0}=0$ and $J_{f}=2 \rightarrow J_{0}=0$ fine-structure transitions, both of which contribute to the $\mathrm{K} \alpha_{1}$ radiation with equal weights. Our effective one-particle calculations were found to be in perfect agreement with the experimental data as well as the previous many-electron calculations.
In the future, we plan to perform a similar angular analysis for the $\mathrm{K} \alpha_{1}$ decay following projectile excitation which also attracts particular experimental interest $[26,40]$. Such an analysis will require an evaluation of the bound-bound transition matrix elements for the Coulomb interaction and, hence, of a new set of alignment parameters. Based again on the effective one-electron approximations, these alignment calculations are currently underway.

The work of U.D.J. was supported by DFG (Heisenberg program). S.F. acknowledges support by BMBF and GSI (project No. KS-FRT).

\section{References}

1. I.C. Percival, M.J. Seaton, Phil. Trans. R. Soc. A 251, 113 (1958)

2. E.G. Berezhko, N.M. Kabachnik, J. Phys. B 10, 2467 (1977)

3. K. Blum, Density Matrix Theory and Applications (Plenum, New York, 1981)

4. V.V. Balashov, A.N. Grum-Grzhimailo, N.M. Kabachnik, Polarization and Correlation Phenomena in Atomic Collisions (Kluwer Academic, New York, 2000)

5. M.K. Inal, J. Dubau, J. Phys. B 20, 4221 (1987)

6. H.L. Zhang, D.H. Sampson, R.E.H. Clark, Phys. Rev. A 41, 198 (1990)

7. K.J. Reed, M.H. Chen, Phys. Rev. A 48, 3644 (1993)

8. S.D. Loch, M.S. Pindzola, C.P. Ballance, D.C. Griffin, J. Phys. B 39, 85 (2006)

9. P. Beiersdorfer, G. Brown, S. Utter, P. Neill, K.J. Reed, A.J. Smith, R.S. Thoe, Phys. Rev. A 60, 4156 (1999)

10. D.L. Robbins, A.Ya. Faenov, T.A. Pikuz, H. Chen, P. Beiersdorfer, M.J. May, J. Dunn, K. J. Reed, A.J. Smith, Phys. Rev. A 70, 022715 (2004)

11. D.L. Robbins, P. Beiersdorfer, A.Ya. Faenov, T.A. Pikuz, D.B. Thorn, H. Chen, K.J. Reed, A.J. Smith, K.R. Boyce, G.V. Brown, R.L. Kelley, C.A. Kilbourne, F.S. Porter, Phys. Rev. A 74, 022713 (2006)

12. G.V. Brown, P. Beiersdorfer, H. Chen, J.H. Scofield, K.R. Boyce, R.L. Kelley, C.A. Kilbourne, F.S. Porter, M.F. Gu, S.M. Kahn, A.E. Szymkowiak, Phys. Rev. Lett. 96, $253201(2006)$

13. S.C. McFarlane, J. Phys. B 5, 1906 (1972)

14. E.G. Berezhko, N.M. Kabachnik, V.V. Sizov, J. Phys. B 11, L421 (1978)

15. H.L. Zhang, D.H. Sampson, M.K. Inal, Phys. Rev. A 63, $052713(1991)$

16. M.K. Inal, H.L. Zhang, D.H. Sampson, C.J. Fontes, Phys. Rev. A 65, 032727 (1992)

17. M.K. Inal, J. Dubau, J. Phys. B 22, 3329 (1989)

18. S. Zakowicz, Z. Harman, N. Grün, W. Scheid, Phys. Rev. A 68, 042711 (2003)

19. X. Ma, P.H. Mokler, F. Bosch, A. Gumberidze, C. Kozhuharov, D. Liesen, D. Sierpowski, Z. Stachura, Th. Stöhlker, A. Warczak, Phys. Rev. A 68, 042712 (2003)

20. A. Kupliauskiene, V. Tutlys, Nucl. Instr. Meth. B 235, 257 (2005)

21. D.A. Church, R.A. Kenefick, D.W. Wang, R.L. Watson, Phys. Rev. A 26, 3093 (1982) 
22. J. Palinkas, G.J. Pedrazzini, D.A. Church, R.A. Kenefick, C.A. Fulton, R.L. Watson, D.W. Wang, Phys. Rev. A 31, $598(1985)$

23. J.H. Scofield, Phys. Rev. A 44, 139 (1991)

24. S. Fritzsche, P. Indelicato, Th. Stöhlker, J. Phys. B 38, S707 (2005)

25. Th. Stöhlker, F. Bosch, A. Gallus, C. Kozhuharov, G. Menzel, P.H. Mokler, H.T. Prinz, J. Eichler, A. Ichihara, T. Shirai, R.W. Dunford, T. Ludziejewski, P. Rymuza, Z. Stachura, P. Swiat, A. Warczak, Phys. Rev. Lett. 79, 3270 (1997)

26. A. Gumberidze, Th. Stöhlker, G. Bednarz, F. Bosch, S. Fritzsche, S. Hagmann, D.C. Ionescu, O. Klepper, C. Kozhuharov, A. Krämer, D. Liesen, X. Ma, R. Mann, P.H. Mokler, D. Sierpowski, Z. Stachura, M. Steck, S. Toleikis, A. Warczak, Hyp. Int. 146/147, 133 (2003)

27. A. Surzhykov, S. Fritzsche, A. Gumberidze, Th. Stöhlker, Phys. Rev. Lett. 85, 153001 (2002)

28. J. Eichler, A. Ichihara, T. Shirai, Phys. Rev. A 58, 2128 (1998)

29. A. Surzhykov, U.D. Jentschura, Th. Stöhlker, S. Fritzsche, Phys. Rev. A 73, 032716 (2006)

30. A. Surzhykov, U.D. Jentschura, Th. Stöhlker, S. Fritzsche, Phys. Rev. A 74, 052710 (2006)
31. E.G. Drukarev, X. Ma, A.I. Mikhailov, I.A. Mikhailov, P.H. Mokler, Phys. Rev. A 74, 022717 (2006)

32. S. Fritzsche, A. Surzhykov, Th. Stöhlker, Phys. Rev. A 72, 012704 (2005)

33. A. Surzhykov, S. Fritzsche, Th. Stöhlker, J. Phys. B 35, $3713(2002)$

34. R. Cowan, The theory of atomic structure and spectra (University of California Press, Berkley, 1981)

35. I.I. Sobel'man, Introduction to the theory of atomic spectra (Pergamon Press, Oxford, 1972)

36. J.H. Scofield, Phys. Rev. A 14, 1418 (1976)

37. S. Fritzsche, J. Electr. Spec. Rel. Phenom. 114-116, 1155 (2001)

38. A. Surzhykov, S. Fritzsche, P. Koval, Comput. Phys. Commun. 165, 139 (2005)

39. W.R. Johnson, D.R. Plante, J. Sapirstein, Adv. At. Mol. Phys. 35, 255 (1995)

40. Th. Stöhlker, D.C. Ionescu, P. Rymuza, F. Bosch, H. Geissel, C. Kozhuharov, T. Ludziejewski, P.H. Mokler, C. Scheidenberger, Z. Stachura, A. Warczak, R.W. Dunford, Phys. Rev. A 57, 845 (1998) 